\title{
ASYMPTOTIC PROPERTIES OF SOLUTIONS OF A CLASS OF IMPULSIVE DIFFERENTIAL EQUATIONS OF SECOND ORDER WITH A RETARDED ARGUMENT
}

\author{
D. D. Bainov, M. B. Dimitrova and A. B. Dishliev
}

\begin{abstract}
Some asymptotic properties are studied for the solutions of a class of impulsive differential equations of second order with retarded argument and fixed moments of impulse effect. Sufficient conditions are found for oscillation of all bounded solutions.
\end{abstract}

\section{Introduction}

The impulsive differential equations are adequate mathematical models of various processes and phenomena studied in physics, chemical technology, population dynamics, technics and economics. That is why, in the recent years they are an object of intensive investigation. Here we mention the monographs [1] and [2], where numerous properties are studied for the solutions of the impulsive differential equations.

The oscillatory theory of the impulsive differential equations is not yet elaborated in contrast to the oscillation theory of ordinary differential equations with deviating argument [4], [5], [6]. The first paper in this area is [3]. Therein sufficient conditions are found for oscillation of all solutions of linear impulsive differential equations of first order with retarded argument and fixed moments of impulse effect. Moreover, conditions on existence of at least one nonoscillatory solution are obtained.

In the present work we study some asymptotic properties of the solutions of a class of impulsive differential equations of second order with retarded argument and fixed moments of impulse effect. Sufficient conditions are found for oscillation of all bounded solutions of the equation under consideration.

AMS (MOS) Subject Classification: 34A37.

Keywords and phrases: Asymptotic properties, impulsive differential equations, retarded argument.

Received August 12, 1996. 


\section{Preliminary notes}

We consider the impulsive differential equation with retarded argument

$$
\begin{aligned}
& \left(r(t) y^{\prime}(t)\right)^{\prime}-f(t, y(t), y(t-h))=0, \quad t \neq \tau_{k}, \quad k \in N \\
& \Delta\left(r\left(\tau_{k}\right) y^{\prime}\left(\tau_{k}\right)\right)=g_{k}\left(y\left(\tau_{k}\right), y\left(\tau_{k}-h\right)\right), \quad \Delta y\left(\tau_{k}\right)=0 .
\end{aligned}
$$

Here $\Delta\left(r\left(\tau_{k}\right) y^{\prime}\left(\tau_{k}\right)\right)=r\left(\tau_{k}+0\right) y^{\prime}\left(\tau_{k}+0\right)-r\left(\tau_{k}-0\right) y^{\prime}\left(\tau_{k}-0\right)$.

We suppose that $y\left(\tau_{k}+0\right)=y\left(\tau_{k}-0\right)=y\left(\tau_{k}\right) ; y^{\prime}\left(\tau_{k}-0\right)=y^{\prime}\left(\tau_{k}\right) ; r\left(\tau_{k}-0\right)=r\left(\tau_{k}\right)$; $h$ is a positive constant, $\tau_{1}, \tau_{2}, \ldots$ are the moments of impulse effect.

We set the next initial conditions for the solutions of (1):

$$
\begin{aligned}
& y(t)=\varphi(t), \quad t \in[-h, 0], \\
& y^{\prime}(0+0)=y_{0}^{\prime}, \quad y(0)=\varphi(0)=y_{0},
\end{aligned}
$$

where $\varphi \in C([-h, 0], \boldsymbol{R})$.

We denote by $P C\left(\overline{\boldsymbol{R}}_{+}, \boldsymbol{R}\right)$ the set of all functions $u: \overline{\boldsymbol{R}}_{+} \rightarrow \boldsymbol{R},\left(\overline{\boldsymbol{R}}_{+}=[0,+\infty)\right)$, which are continuous for $t \in \overline{\boldsymbol{R}}_{+}, t \neq \tau_{k}, k \in \boldsymbol{N}$, continuous from the left for $t \in \overline{\boldsymbol{R}}_{+}$ and having discontinuity of the first kind at the points $\tau_{k} \in \overline{\boldsymbol{R}}_{+}, k \in \boldsymbol{N}$.

We denote by $P C\left(\overline{\boldsymbol{R}}_{+}, \boldsymbol{R}\right)$ the set of all functions $u: \overline{\boldsymbol{R}}_{+} \rightarrow \boldsymbol{R}$, for which $(d u / d t) \in P C\left(\overline{\boldsymbol{R}}_{+}, \boldsymbol{R}\right)$.

We introduce the following conditions:

H1. $f \in C\left(\overline{\boldsymbol{R}}_{+} \times \boldsymbol{R}^{2}, \boldsymbol{R}\right)$, the function $f(t, u, v)$ is nondecreasing with respect to $u$ and $v$ for each fixed $t \geqq 0$, and there exists a constant $T>0$ such that $u f(t, u, v)>0$ for $u v>0$ and $t \geqq T$.

H2. $g_{k} \in C\left(\boldsymbol{R}^{2}, \boldsymbol{R}\right), k \in \boldsymbol{N}, g_{k}(u, v)$ are nondecreasing functions with respect to $u$ and $v, u g_{k}(u, v)>0$ for those $k$ for which $\tau_{k} \geqq T$ and $u v>0, k \in N$.

H3. $r \in P C\left(\boldsymbol{R}_{+}, \boldsymbol{R}_{+}\right), r\left(\tau_{k}+0\right)>0$ for $\tau_{k} \in \boldsymbol{R}_{+}=(0,+\infty)$.

H4. $0<\tau_{1}<\tau_{2}<\cdots, \lim _{k \rightarrow \infty} \tau_{k}=+\infty$.

DEFINITION 1. Solution of the equation (1) with initial conditions (2) will be called any function $y:[-h, \infty) \rightarrow \boldsymbol{R}$ for which the following conditions are fulfilled :

1. If $-h \leqq t \leqq 0$, then $y(t)=\varphi(t)$.

2 . If $0<t \leqq \tau_{1}$, then the solution $y$ coincides with the solution of the problem (1), (2) without impulse effect.

3. If $\tau_{k}<t \leqq \tau_{k+1}, k \in N$, the solution of the problem (1), (2) coincides with the solution of the integro-differential equation

$$
\begin{aligned}
r(t) y^{\prime}(t) & =r\left(\tau_{k}+0\right) y^{\prime}\left(\tau_{k}+0\right)+\int_{\tau_{k}}^{t} f(s, y(s), y(s-h)) d s \\
& =r\left(\tau_{k}\right) y^{\prime}\left(\tau_{k}\right)+g_{k}\left(y\left(\tau_{k}\right), y\left(\tau_{k}-h\right)\right)+\int_{\tau_{k}}^{t} f(s, y(s), y(s-h)) d s
\end{aligned}
$$

with initial conditions (2). 
DEFinition 2. The solution $y$ of the problem (1) is said to be oscillatory if for each $a>0$ we have

$$
\{t: y(t)>0, t>a\} \neq \emptyset, \quad\{t: y(t)<0, t>a\} \neq \emptyset .
$$

Otherwise, the solution $y$ is called nonoscillatory.

Let $S$ denote the set of all solutions of the equation (1). We introduce the following sets :

$$
\begin{aligned}
& S^{+\infty}=\left\{y \in S: \lim _{t \rightarrow+\infty} y(t)=+\infty, \lim _{t \rightarrow+\infty} r(t) y^{\prime}(t)=+\infty\right\}, \\
& S^{-\infty}=\left\{y \in S: \lim _{t \rightarrow+\infty} y(t)=-\infty, \lim _{t \rightarrow+\infty} r(t) y^{\prime}(t)=-\infty\right\}, \\
& S^{k}=\left\{y \in S: 0<\lim _{t \rightarrow+\infty} y(t)<+\infty, \lim _{t \rightarrow+\infty} r(t) y^{\prime}(t)=0\right\}, \\
& S^{-k}=\left\{y \in S:-\infty<\lim _{t \rightarrow+\infty} y(t)<0, \lim _{t \rightarrow+\infty} r(t) y^{\prime}(t)=0\right\}, \\
& S^{0}=\left\{y \in S: \lim _{t \rightarrow+\infty} y(t)=0, \lim _{t \rightarrow+\infty} r(t) y^{\prime}(t)=0\right\}, \\
& S^{\sim}=\{y \in S: y(t) \text { is oscillatory solution }\} .
\end{aligned}
$$

\section{Main results}

THEOREM 1. Let the following conditions be fulfilled:

1. Conditions $\mathrm{H} 1-\mathrm{H} 4$ are met.

2. $\int^{\infty} \frac{d t}{r(t)}=+\infty$.

3. $\int_{T}^{\infty}|f(s, c R(s), c R(s-h))| d s+\sum_{\tau_{k} \geq T}\left|g_{k}\left(c R\left(\tau_{k}\right), c R\left(\tau_{k}-h\right)\right)\right|=+\infty$,

where $R(t)=\int_{0}^{t} \frac{d u}{r(u)}$ for some nonzero constant $c$.

Then

$$
S=S^{+\infty} \cup S^{-\infty} \cup S^{k} \cup S^{-k} \cup S^{0} \cup S^{\sim}
$$

Proof. Let $y \in S \backslash S^{\sim}$. The following two cases are possible:

Case 1. $y(t)>0$ for $t \geqq t_{1} \geqq T$. Then, it follows from conditions $\mathrm{H} 1$ and $\mathrm{H} 2$ that there exists a point $t_{2} \geqq t_{1}$ such that $\Delta\left(r\left(\tau_{k}\right) y^{\prime}\left(\tau_{k}\right)\right)>0$ and $\left(r(t) y^{\prime}(t)\right)^{\prime}>0$ for $t, \tau_{k} \geqq t_{2}$. Therefore, there exists a point $t_{3} \geqq t_{2}$ such that $r(t) y^{\prime}(t)$ has a constant 
sign for $t \geqq t_{3}$.

Let $r(t) y^{\prime}(t) \geqq c>0$ for $t \geqq t_{3}$. We shall prove that $y \in S^{+\infty}$.

Since $y^{\prime}(t) \geqq c / r(t), t \geqq t_{3}$, it follows

$$
y(t) \geqq y\left(t_{3}\right)+c \int_{t_{3}}^{t} \frac{d u}{r(u)} .
$$

The last inequality and condition 2 yield $\lim _{t \rightarrow+\infty} y(t)=+\infty$ and

$$
\frac{y(t)}{R(t)} \geqq c \frac{R(t)-R\left(t_{3}\right)}{R(t)} .
$$

Let us choose $t_{4}\left(t_{4} \geqq t_{3}\right)$ such that $R\left(t_{4}\right) \geqq 2 R\left(t_{3}\right)$. Then, it follows from (3) for $t \geqq t_{4}$ that

$$
\frac{y(t)}{R(t)} \geqq \frac{c}{2}=c_{1}
$$

i.e.,

$$
y(t) \geqq c_{1} R(t) \text { and } \quad y(t-h) \geqq c_{1} R(t-h) \quad \text { for } t \geqq t_{5}=t_{4}+h .
$$

Now, integrating (1) from $t_{5}$ to $t$ and taking into account the monotonicity of the functions $f$ and $g_{k}(k \in \boldsymbol{N})$ we arrive at the inequality

$$
\begin{aligned}
r(t) y^{\prime}(t) \geqq & r\left(t_{5}\right) y^{\prime}\left(t_{5}\right)+\int_{t_{5}}^{t} f\left(s, c_{1} R(s), c_{1} R(s-h)\right) d s \\
& +\sum_{t_{5} s \tau_{k}<t} g_{k}\left(c_{1} R\left(\tau_{k}\right), c_{1} R\left(\tau_{k}-h\right)\right) .
\end{aligned}
$$

It follows from the above inequality as $t \rightarrow+\infty$, and from condition 3 that

$$
\lim _{t \rightarrow+\infty} r(t) y^{\prime}(t)=+\infty \text {. }
$$

Let $r(t) y^{\prime}(t)<0, t \geqq t_{3}$. We shall prove that $y \in S^{0} \cup S^{k}$.

Since $y(t)>0, y^{\prime}(t)<0, r(t) y^{\prime}(t)<0$ and $r(t) y^{\prime}(t)$ is an increasing function for $t \geqq t_{3}$, then there exist the finite limits

$$
\lim _{t \rightarrow+\infty} y(t)=y(+\infty) \geqq 0 \text { and } \lim _{t \rightarrow+\infty} r(t) y^{\prime}(t)=L \leqq 0 .
$$

Let us suppose $\lim _{t \rightarrow+\infty} r(t) y^{\prime}(t)=L<0$. Then $r(t) y^{\prime}(t)<L$ for $t \geqq t_{3}$. Therefore,

$$
y(t) \leqq y\left(t_{3}\right)+L \int_{t_{3}}^{t} \frac{d u}{r(u)}
$$

It follows from (4) after passing to limit as $t \rightarrow+\infty$ that $\lim _{t \rightarrow+\infty} y(t)=-\infty$, which contradicts the assumption that $y$ is a positive solution. Therefore, 


$$
\lim _{t \rightarrow+\infty} r(t) y^{\prime}(t)=0 \text {. }
$$

It follows from (5) that either $\lim _{t \rightarrow+\infty} y(t)=0$, or $\lim _{t \rightarrow+\infty} y(t)=k(0<k<+\infty)$, i.e., $y \in S^{0} \cup S^{k}$.

Case 2. In the case when $y$ is a negative solution of the equation (1), the proof is analogous to this in case 1 .

THEOREM 2. Let the following conditions be fulfilled:

1. Conditions $\mathrm{H} 1-\mathrm{H} 4$ are met.

2. $\int^{\infty} \frac{d s}{r(s)}=+\infty$ and $r^{\prime}(t) \geqq 0$ for $t \in \boldsymbol{R}_{+}$.

3. There exist function $p(t)$ and sequence $\left\{\beta_{k}\right\}_{k=1}^{\infty}$ such that $p \in P C\left(\overline{\boldsymbol{R}}_{+}, \boldsymbol{R}_{+}\right)$ and $\beta_{k} \geqq 0, k \in \boldsymbol{N}$.

4. The inequalities $v f(t, u, v) \geqq v^{2} p(t)$ and $v g_{k}(u, v) \geqq v^{2} \beta_{k}$ for $u v>0, k \in \boldsymbol{N}$ are fulfilled.

5. $\quad \limsup _{t \rightarrow+\infty} \frac{1}{r(t)}\left[\int_{t-h}^{t}(u-t+h) p(u) d u+\sum_{t-h \leqq \tau_{k}<t}\left(\tau_{k}-t+h\right) \beta_{k}\right]>1$.

Then each bounded solution of the equation (1) is oscillatory.

Proof. Let $y(t)$ be a nonoscillatory bounded solution of the equation (1). Without loss of generality we may suppose that $y(t)>0$ for $t \geqq t_{1} \geqq 0$ and $y(t) \leqq M$, $M=$ const $>0$. It is clear, that $y(t-h)>0$ for $t \geqq t_{2}=t_{1}+h$.

It follows from conditions $\mathrm{H} 1, \mathrm{H} 2$ and from $(1)$ that $\left(r(t) y^{\prime}(t)\right)^{\prime}>0$ and $\Delta\left(r\left(\tau_{k}\right) y^{\prime}\left(\tau_{k}\right)\right)>0$ for $t, \tau_{k} \geqq t_{2}$, i.e., $r(t) y^{\prime}(t)$ is an increasing function for $t \geqq t_{2}$.

The next cases are possible:

Case 1. $\quad r(t) y^{\prime}(t) \geqq c>0$ for $t \geqq t_{2}$. Analogously to the proof of Theorem 1 we obtain the equality $\lim _{t \rightarrow+\infty} y(t)=+\infty$, which contradicts the boundedness of the solution $y$.

Case 2. $r(t) y^{\prime}(t) \leqq 0, t \geqq t_{2}$. Therefore, $y(t)$ is nonincreasing function for $t \geqq t_{2}$. Then, in view of condition 4 for $t \geqq t_{3}=t_{2}+h$ we arrive at

$$
\begin{aligned}
& \left(r(t) y^{\prime}(t)\right)^{\prime} \geqq p(t) y(t-h), \quad t \neq \tau_{k}, \quad k \in \boldsymbol{N} \\
& \Delta\left(r\left(\tau_{k}\right) y^{\prime}\left(\tau_{k}\right)\right) \geqq \beta_{k} y\left(\tau_{k}-h\right), \quad \Delta y\left(\tau_{k}\right)=0 .
\end{aligned}
$$

Integrating (6) from $s$ to $t\left(t_{3} \leqq s<t\right)$, we obtain

$$
0 \geqq r(t) y^{\prime}(t) \geqq r(s) y^{\prime}(s)+\sum_{s \leq \tau_{k}<t} \beta_{k} y\left(\tau_{k}-h\right)+\int_{s}^{t} p(u) y(u-h) d u .
$$

We integrate again the above inequality from $t-h$ to $t$ and arrive at the inequality 


$$
0 \geqq \int_{t-h}^{t} r(s) y^{\prime}(s) d s+\int_{t-h}^{t}\left[\int_{s}^{t} p(u) y(u-h) d u+\sum_{s \leq \tau_{k}<t} \beta_{k} y\left(\tau_{k}-h\right)\right] d r .
$$

Changing the order of integration in (7) implies

$$
\begin{aligned}
0 \geqq & \int_{t-h}^{t} r(s) y^{\prime}(s) d s+\int_{t-h}^{t}(u-t+h) p(u) y(u-h) d u \\
& +\sum_{t-h \leq \tau_{k}<t}\left(\tau_{k}-t+h\right) \beta_{k} y\left(\tau_{k}-h\right) .
\end{aligned}
$$

Now, from the fact that $r(t)$ is nondecreasing function in $\overline{\boldsymbol{R}}_{+}$and $y(t)$ is nonincreasing function for $t \geqq t_{2}$, as well as from (8) it follows the inequality

$$
\begin{aligned}
& 0 \geqq r(t-h)[y(t)-y(t-h)] \\
& \quad+y(t-h)\left[\int_{t-h}^{t}(u-t+h) p(u) d u+\sum_{t-h \leqq \tau_{k}<t}\left(\tau_{k}-t+h\right) \beta_{k}\right]
\end{aligned}
$$

which means

$$
\begin{aligned}
& y(t-h)\left[\int_{t-h}^{t}(u-t+h) p(u) d u+\sum_{t-h \leqq \tau_{k}<t}\left(\tau_{k}-t+h\right) \beta_{k}\right] \\
\leqq & r(t-h) y(t-h) \leqq r(t) y(t-h),
\end{aligned}
$$

i.e.,

$$
\frac{1}{r(t)}\left[\int_{t-h}^{t}(u-t+h) p(u) d u+\sum_{t-h \leqq \tau_{k}<t}\left(\tau_{k}-t+h\right) \beta_{k}\right] \leqq 1 .
$$

The last inequality contradicts condition 5 of Theorem 2 .

If $-M \leqq y(t)<0$ for $t \geqq t_{1} \geqq 0$, analogous arguments as above lead to a contradiction with condition 5 of Theorem 2 .

Let us consider now the following equation which is a particular variant of the equation (1):

$$
\begin{aligned}
& y^{\prime \prime}(t)-a(t) y(t)-b(t) y(t-h)=0, \quad t \neq \tau_{k}, \quad k \in N \\
& \Delta y^{\prime}\left(\tau_{k}\right)=a_{k}\left(\tau_{k}\right) y\left(\tau_{k}\right)+b_{k}\left(\tau_{k}\right) y\left(\tau_{k}-h\right), \quad \Delta y\left(\tau_{k}\right)=0 .
\end{aligned}
$$

We introduce the next condition:

H5. $a, b, a_{k}, b_{k} \in C\left(\overline{\boldsymbol{R}}_{+}, \boldsymbol{R}_{+}\right), k \in \boldsymbol{N}$.

COROLLARY 1. Let the following conditions be fulfilled:

1. Conditions $\mathrm{H} 4$ and $\mathrm{H} 5$ are fulfilled.

2. $\quad \limsup _{t \rightarrow+\infty}\left[\int_{t-h}^{t}(u-t+h) b(u) d u+\sum_{t-h \leqq \tau_{k}<t}\left(\tau_{k}-t+h\right) b_{k}\left(\tau_{k}\right)\right]>1$.

Then each bounded solution of the equation (9) is oscillatory. 
Proof. In this case,

$$
\begin{aligned}
& f(t, y(t), y(t-h))=a(t) y(t)+b(t) y(t-h) \\
& g_{k}\left(y\left(\tau_{k}\right), y\left(\tau_{k}-h\right)\right)=a_{k}\left(\tau_{k}\right) y\left(\tau_{k}\right)+b_{k}\left(\tau_{k}\right) y\left(\tau_{k}-h\right) \\
& p(t)=b(t), \quad \beta_{k}=b_{k}\left(\tau_{k}\right), \quad r(t) \equiv 1 .
\end{aligned}
$$

A straightforward verification shows that conditions $\mathrm{H} 1, \mathrm{H} 2$, as well as conditions 2, 3 and 4 of Theorem 2 are fulfilled. Therefore, in view of Theorem 2 each bounded solution of the equation (9) is oscillatory.

Acknowledgement. The present investigation was supported by the Bulgarian Ministry of Education, Science and Technologies under Grant MM-511.

\section{REFERENCES}

[1] D. D. Bainov, V. Lakshmikantham and P.S. Simeonov, Theory of Impulsive Differential Equations, World Scientific, Singapore, 1989.

[2] D.D. Bainov AND P.S. Simeonov, Systems with Impulse Effect, Stability, Theory and Applications, Ellis Horwood, Chichester, 1989.

[3] K. Gopalsamy and B.G. Zhang, On delay differential equations with impulses, J. Math. Anal. Appl., 139 (1989), 110-122.

[4] I. GYöri AND G. LADAs, Oscillation Theory of Delay Differential Equations with Applications, Clarendon Press, Oxford, 1991.

[5] G.S. LAdDE, V. LAKShmikantham and B.G. Zhang, Oscillation Theory of Differential Equations with Deviating Arguments, Pure and Applied Mathematics, 110, Marcel Dekker, New York, 1987.

[6] V.N. Shevelo, Oscillations of Solutions of Differential Equations with Deviating Arguments, Naukova Dumka, Kiev, 1978 (in Russian).

Medical University of Sofia

P.O. BOX 45

1504 SOFIA

BULGARIA

TECHNICAL UNIVERSITY

SLiven, Bulgaria

University of Chemical Technologies and Metallurgy

Sofia, Bulgaria 